\title{
Hemorragia retroperitoneal espontánea: nuestra experiencia en los últimos 10 años
}

\author{
Gimeno Argente V, Bosquet Sanz M, Ramírez Backhaus M, Trassierra Villa M, \\ Arlandis Guzmán S, Jiménez Cruz JF.
}

Servicio de Urología. Hospital Universitario La Fe. Valencia.

Actas Urol Esp. 2007;31(5):521-527

\section{RESUMEN}

HEMORRAGIA RETROPERITONEAL ESPONTÁNEA: NUESTRA EXPERIENCIA EN LOS ÚLTIMOS 10 AÑOS

Introducción y Objetivos: $\mathrm{El}$ análisis de la etiología, manifestaciones clínicas, métodos diagnósticos y tratamiento empleado en la hemorragia retroperitoneal espontánea (HRE), en nuestra serie de pacientes.

Métodos: Presentamos 27 casos de HRE diagnosticados en nuestro hospital entre Enero de 1996 y Diciembre de 2005. Las técnicas de imagen empleadas fueron ecografia abdominal, TC abdomino-pélvica y resonancia magnética.

Resultados: La causa más frecuente de sangrado fue la rotura de un angiomiolipoma renal en 7 pacientes. Entre las manifestaciones clínicas, el dolor lumbar o abdominal fue el síntoma predominante. La ecografía abdominal detectó el hematoma en el $81,8 \%$ de pacientes, aportando un diagnóstico etiológico en tan sólo el 40,9\% de los casos. La TC abdomino-pélvica reveló la hemorragia retroperitoneal en todos casos, y además diagnosticó en el 92,6\%, el origen del sangrado. El tratamiento fue cirugía de urgencia en 10 pacientes, mientras que en los 17 restantes se optó, inicialmente, por medidas terapéuticas conservadoras.

Conclusiones: En nuestra experiencia, en los casos de HRE, la TC abdomino-pélvica es la prueba diagnóstica de elección y el manejo terapéutico de esta patología deberá individualizarse en función de la situación hemodinámica del paciente y la etiología del síndrome.

Palabras clave: Hemorragia retroperitoneal espontánea. Sindrome de Wünderlich. Tratamiento conservador.

\section{ABSTRACT}

SPONTANEOUS RETROPERITONEAL HEMORRHAGE: OUR EXPERIENCE AT LAST 10 YEARS

Introduction and Objectives: Etiology, clinical features, diagnostic methods and treatment of spontaneous retroperitoneal hemorrhage were analyzed.

Methods: We report 27 cases with diagnosis of spontaneous retroperitoneal hemorrhage treated in our hospital between January 1996 and December 2005. The imaging techniques were abdominal ultrasonography, abdominal CT scan and MRI.

Results: The most common cause of retroperitoneal hemorrhage was renal angiomyolipoma rupture in 7 patients. Continuous flank or abdominal pain were the primary symptoms. Abdominal ultrasonography showed hematoma in $81.8 \%$ patients, but the actual etiologic diagnosis was ascertained in only $40.9 \%$ of them. Retroperitoneal hemorrhage was demonstrated by means of abdominal CT scan in all cases and bleeding origin was established in $92.6 \%$ of cases. Ten patients underwent urgent surgery while conservative treatment was attempted in the remaining 17.

Conclusions: In our experience, in cases of spontaneous retroperitoneal hemorrhage, CT scan is the best imaging method to establish the diagnosis and the management of such entity although it will need to be individualized for every case because it depends on the hemodinamic situation and etiologic diagnosis.

Keywords: Spontaneous retroperitoneal hemorrhage. Wünderlich syndrome. Conservative treatment. 
$\mathrm{L}$ a hemorragia retroperitoneal espontánea (HRE) engloba a toda extravasación de sangre al espacio retroperitoneal sin que exista traumatismo externo, manipulación endourológica o endovascular previa. Es una entidad de etiología múltiple (Tabla 1), destacando la rotura de un aneurisma de aorta abdominal como causa más frecuente en adultos ${ }^{1-4}$. A diferencia de hemorragias de otras localizaciones, el sangrado retroperitoneal puede pasar desapercibido en un primer momento, lo que entraña una gran dificultad diagnóstica. Sin embargo, un retraso en el diagnóstico y manejo de esta patología suele traducirse en una elevada morbi-mortalidad, al tratarse de situaciones clínicas graves ${ }^{5}$.

Tabla 1

Etiologia de la HRE. (Modificado de Machuca Santacruz J. et al.)

\section{CAUSAS LOCALES}

\section{Rotura de aneurisma de aorta abdominal}

2. Origen Renal:

Tumores:

- Benignos: Angiomiolipoma

- Malignos: Adenocarcinoma

Enfermedades vasculares:

- Aneurisma de arteria renal

- Trombosis, infartos

- Fístulas arteriovenosas

Otras:

- Infecciones (abscesos, pielonefritis, tuberculosis)

- Nefritis: aguda, crónica

- Nefroesclerosis

- Rechazo agudo o crónico de trasplante renal

3. Origen Suprarrenal:

Tumores:

- Benignos: Feocromocitoma, adenoma, mielolipoma

- Malignos: Feocromocitoma, metástasis, carcinoma Situaciones de estrés :

- Sepsis, cirugía, grandes quemados, traumatismos Tratamientos prolongados con corticoides y ACTH Apoplejía adrenal idiopática

4. Organos Retroperitoneales:

Páncreas

Tumores retroperitoneales

Vasos retroperitoneales

\section{CAUSAS SISTÉMICAS}

1. Alteraciones de la coagulación:

Terapia anticoagulante o antiagregante

Discrasias sanguíneas:

- Leucemia, policitemia, hemofilia, púrpura trombopénica

Hemodiálisis

2. Vasculitis
Presentamos nuestra experiencia en esta patología durante los últimos 10 años, analizando la actitud diagnóstica y terapéutica en nuestra serie.

\section{MATERIAL Y MÉTODOS}

Realizamos un estudio retrospectivo entre Enero de 1996 y Diciembre de 2005, que incluyó a 27 pacientes (17 hombres y 10 mujeres) diagnosticados de HRE en nuestro hospital, con edades comprendidas entre los 22 y 85 años (edad media de 62,9 años).

A todos los pacientes se efectuó una anamnesis y exploración física detallada, así como una analítica sanguínea que incluía hemograma, bioquímica básica y coagulación. Las pruebas de imagen empleadas fueron la radiografia simple de abdomen, ecografía abdominal, tomografia computerizada (TC) abdomino-pélvica y resonancia magnética (RM) en aquellos casos de diagnóstico etiológico incierto mediante TC.

En aquellos pacientes donde la situación hemodinámica fue inestable, se realizó reposición de la volemia con fluidoterapia y/o hemoderivados como medida terapéutica inicial, seguida de intervención quirúrgica urgente si persistía la inestabilidad hemodinámica o existía diagnóstico de certeza con indicación de cirugía.

\section{RESULTADOS}

La etiología de la HRE en los pacientes de nuestra serie queda reflejada en la Tabla 2. Los angiomiolipomas renales fueron la causa más frecuente de esta patología, englobando más del $25 \%$ de los casos.

Tabla 2

Etiología de la HRE en nuestra serie

\begin{tabular}{lc}
\hline & $\mathbf{n}(\%)$ \\
\hline Angiomiolipoma renal & $7(25,9)$ \\
Aneurisma aorta abdominal & $6(22,2)$ \\
Terapia anticoagulante & $5(18,5)$ \\
Tumores malignos & $3(11,1)$ \\
Discrasias sanguíneas & $2(7,4)$ \\
Aneurisma de arteria renal & $1(3,7)$ \\
Absceso renal & $1(3,7)$ \\
Litiasis renoureteral & $1(3,7)$ \\
Idiopática & $1(3,7)$ \\
Total & $27(100)$ \\
\hline
\end{tabular}


Entre las manifestaciones clínicas (recogidas en la Tabla 3), el dolor lumbar o abdominal fue el sintoma predominante, seguido de otros signos como shock hipovolémico y tumoración en flanco. La asociación de estas manifestaciones conforman la denominada triada de Lenk, estando presente en sólo $4(14,8 \%)$ de nuestros pacientes.

Tabla 3

Manifestaciones clinicas

\begin{tabular}{lc}
\hline & n (\%) \\
\hline Dolor lumbar o abdominal & $18(66,7)$ \\
Anemia & $15(55,5)$ \\
Leucocitosis & $14(51,8)$ \\
Shock hipovolémico & $8(29,6)$ \\
Triada de Lenk & $4(14,8)$ \\
Masa palpable & $3(11,1)$ \\
Hematuria & $2(7,4)$ \\
Fiebre & $2(7,4)$ \\
\hline
\end{tabular}

El tiempo medio transcurrido desde el inicio de las manifestaciones clínicas hasta que nos consultaron fue de 21,6 horas.

Entre las pruebas radiológicas de imagen empleadas para el diagnóstico de la HRE, la ecografia abdominal detectó el hematoma en 18 de los 22 pacientes a los que se les efectuó, es decir en el $81,8 \%$ de ellos, diagnosticando la causa del sangrado en tan sólo $9(40,9 \%)$ de los casos. La TC abdomino-pélvica se realizó a todos los pacientes, detectando la hemorragia retroperitoneal en los 27 casos. Además fue capaz de diagnosticar en 25 de ellos $(92,6 \%)$, el origen del sangrado. En las 2 situaciones restantes de diagnóstico etiológico incierto mediante TC, empleamos la RM abdominal siendo diagnóstica en ambos casos (un adenocarcinoma y un angiomiolipoma renal).

El tratamiento fue inicialmente conservador en 17 pacientes (63\%). De éstos, en 2 se realizó embolización arterial selectiva, en 6 se practicó cirugía diferida (3 nefrectomías simples, 1 nefroureterectomía, 1 tumorectomía bilateral y 1 adrenalectomía) y en los 9 restantes se llevó cabo un tratamiento exclusivamente médico dirigido a conseguir y/o preservar la estabilidad hemodinámica. En los otros 10 casos, se efectuó cirugía de urgencia $(6$ by-pass aorto-bifemoral, 1 reparación de arteria renal, 2 nefrectomías simples, 1 evacuación y drenaje del hematoma).
La evolución de los pacientes que recibieron tratamiento inicialmente conservador fue satisfactoria en el 82,4\% (14/17). Al analizar la evolución de nuestros pacientes en función de la etiología del sangrado, obtuvimos que aquellas hemorragias de origen renal evolucionaron de forma satisfactoria en el 83,3\% (10/12) de casos, falleciendo 2 de ellos por un shock séptico al undécimo día de una nefrectomía radical por adenocarcinoma y por un shock hipovolémico en las primeras 24 horas tras la reparación quirúrgica de una rotura de aneurisma de arteria renal. Sin embargo, de los 15 casos de HRE de causa extrarrenal, el porcentaje de fallecimientos se situó en el 46,7\% (7/15), teniendo que considerar la patologia de base que presentaban todos ellos (3 aneurismas de aorta abdominal, 2 discrasias sanguíneas severas y 2 arritmias cardiacas graves en tratamiento anticoagulante), como factor determinante en su pronóstico.

La mortalidad global en nuestra serie ascendió al 30\% (9/27). De los que fallecieron, el $44,4 \%$ (4/9) lo hicieron en el quirófano de urgencia, debido a causas vasculares como rotura de aneurisma de aorta abdominal (3) o de arteria renal (1). El resto de fallecimientos acontecieron durante la primera semana de evolución, lo que indica que una vez superado este periodo de tiempo aumenta en gran medida las posibilidades de supervivencia.

\section{DISCUSIÓN}

La hemorragia retroperitoneal espontánea es un síndrome clínico infrecuente, de etiología multifactorial. Como mencionamos con anterioridad, la rotura de un aneurisma de aorta abdominal supone la mayoría de casos de esta patología (Fig. 1). Sin embargo, las causas que más nos interesan desde el punto de vista urológico son las de origen renal y suprarrenal, al igual que aquellas sistémicas.

La HRE de origen renal fue observada por primera vez en 1700 por Bonet, siendo Wünderlich quien la define como "apoplejía espontánea de la capsula renal" en 1856. Con posterioridad, Coenen en 1910 designa a esta entidad con el término de "Síndrome de Wünderlich"6-12. La mayoría de autores coinciden en señalar que la causa principal de estas hemorragias son los tumores 


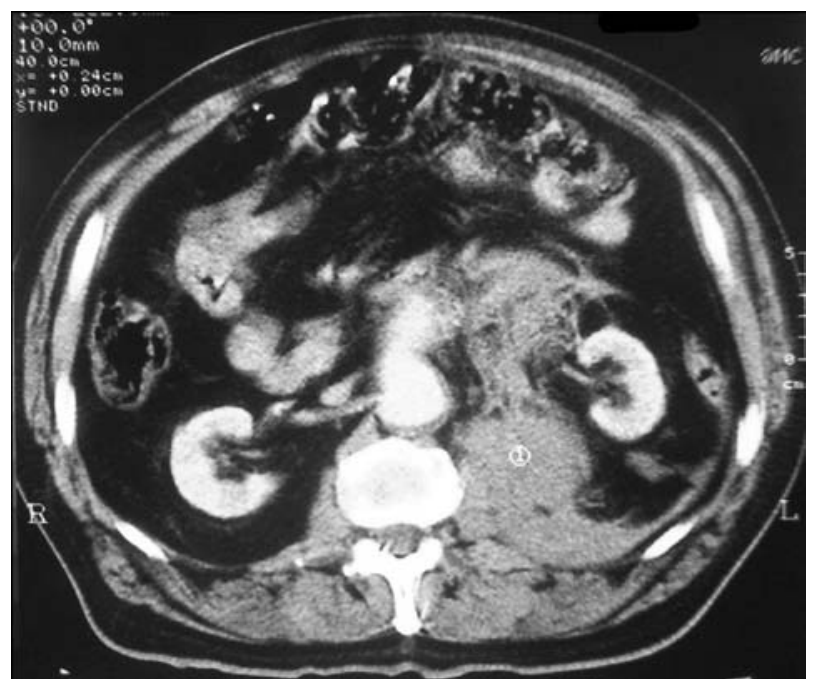

FIGURA 1. Imagen de TC abdómino-pélvica con contraste que muestra rotura de aneurisma de aorta abdominal infrarrenal, con colección hemática en espacio perirrenal izquierdo.

renales (entre el 57 y 63\%), seguidas de las enfermedades vasculares (18-26\%) y las inflamatorias $(11 \%)^{13-16}$. Entre los tumores renales, la mitad de los casos presentan una histología benigna, siendo el angiomiolipoma (AML) el responsable de la mayoría de HRE de este grupo (Fig. 2). Aproximadamente el $15 \%$ de los AML debutan con esta forma de presentación ${ }^{17}$, en especial los mayores de $4 \mathrm{~cm}$ que llegan a presentar en el $51 \%$ de casos una HRE en algún momento ${ }^{18}$. El adenocarcinoma renal es el tumor maligno que con mayor frecuencia produce HRE, aunque su incidencia de rotura es muy baja (0,3-1,3\%). Con menor frecuencia se han descrito otros tumores malignos causantes de este síndrome como tumor de Wilms, carcinoma transicional, sarcoma o metástasis renales de otros tumores ${ }^{3,9}$. Las enfermedades vasculares responsables de HRE son raras, destacando el aneurisma de arteria renal como causa principal, al alcanzar entre el 5 y $10 \%$ de todas las $\mathrm{HRE}^{14,16}$. Las infecciones del parénquima renal (pielonefritis, abscesos, tuberculosis, etc.), enfermedades quísticas, hidronefrosis o litiasis, son otras causas menos frecuentes de $\mathrm{HRE}^{3}$. En nuestra serie, los 12 casos de hemorragia de origen renal se debieron a 7 AML (58,3\%), 1 adenocarcinoma, 1 carcinoma transicional de pelvis renal, 1 aneurisma de arteria renal, 1 absceso renal y 1 litiasis ureteral.
El origen suprarrenal de la HRE es poco frecuente y se asocia a situaciones de estrés severo (sepsis, cirugia, grandes quemados, gestaciones complicadas) y tratamientos prolongados con corticoides o ACTH, siendo el sangrado en estos casos bilateral. Cuando se detectan hematomas unilaterales, se suelen deber a causas locales como quistes, tumores (feocromocitoma, adenoma, carcinoma, mielolipoma y lesiones metastásicas) o en ocasiones idiopáticas ${ }^{2,4}$. En uno de nuestros pacientes una metástasis suprarrenal de un carcinoma epidermoide de pulmón provocó un hematoma retroperitoneal.

De las causas sistemicas, la panarteritis nodosa es la más frecuente, registrando hasta un $22 \%$ en algunas series ${ }^{19}$. Se trata de una vasculitis autoinmune por depósito de inmunocomplejos en las paredes de arterias de pequeño y mediano calibre. Estos depósitos destruyen la capa media arterial causando trombosis y aneurismas, con la consiguiente fragilidad arterial. La afectación renal se sitúa entorno al $80 \%$ de los $\operatorname{casos}^{1,4,20,21}$. Otras causas sistémicas son los tratamientos con anticoagulantes y antiagregantes plaquetarios, las discrasias sanguíneas (leucemia, policitemia, trombopenia, hemofilia, mieloma,...) y la hemodiálisis. De nuestros pacientes, en 7 casos se registró un origen sistémico, 4 recibieron terapia anticoagulante oral (acenocumarol), 1 antiagregantes plaquetarios (clopidogrel) y 2 presentaban discrasias sanguíneas en forma de mieloma múltiple y trombopenia idiopática.

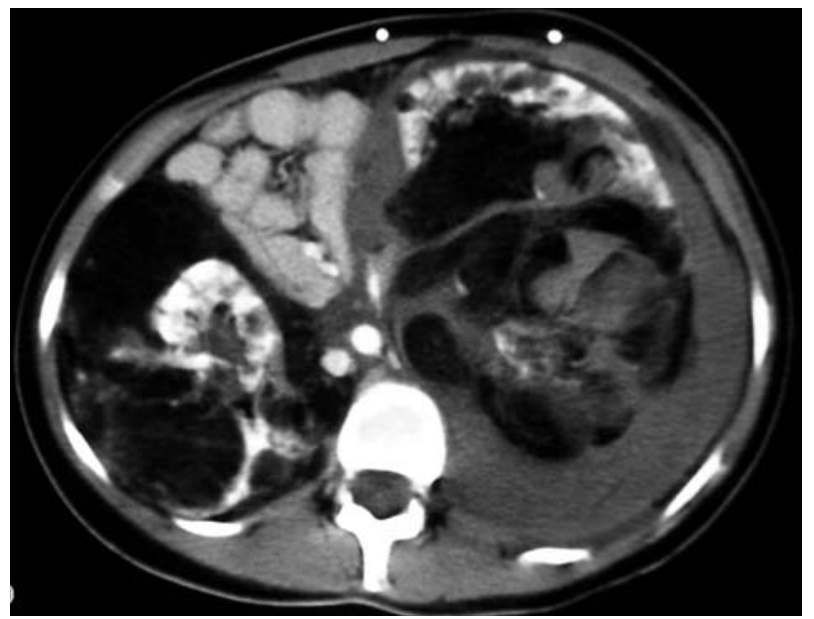

FIGURA 2. Imagen de TC abdómino-pélvica donde se observa un hematoma perirrenal derecho $y$ otro intrarrenal izquierdo, ambos provocados por rotura de angiomiolipomas renales bilaterales. 
El diagnóstico de este síndrome se basará en la sintomatología clínica, exploración física, datos analíticos y estudio radiológico.

La presentación clínica de la HRE dependerá de la intensidad y duración del sangrado. No existen signos o síntomas patognomónicos, siendo el dolor lumboabdominal el síntoma principal. En ocasiones se asocia a la existencia de masa palpable en flanco $\mathrm{y}$, cuando el sangrado es importante, a signos de hipovolemia como sudoración profusa, palidez y frialdad cutánea, hipotensión y shock. Esta triada (dolor lumboabdominal, masa palpable y shock hipovolémico) fue descrita inicialmente por Lenk, estando presente entorno al 30\% de los casos ${ }^{2}$. En nuestra casuística, el dolor lumbar o abdominal fue el síntoma predominante $(66,7 \%)$, pero tan solo 7 pacientes (14,8\%) presentaron la triada de Lenk. Sin embargo, la mayoría de hemorragias suelen ser insidiosas y progresivas por lo que los síntomas se desarrollarán gradualmente durante días, dificultando en gran medida el diagnóstico. Con menos frecuencia podemos encontrar hematuria, fiebre, nauseas y vómitos o signos de irritación peritoneal.

Los parámetros analíticos más relevantes son el descenso de hemoglobina y hematocrito, la leucocitosis y el aumento de las cifras de VSG, LDH, urea y creatinina ${ }^{1}$.

Las pruebas radiológicas actuales deben ser el pilar diagnóstico de esta patología, no sólo detectando el hematoma retroperitoneal sino aportando un diagnóstico etiológico que nos permita evitar exploraciones quirúrgicas innecesarias. Clásicamente, la radiografía simple de abdomen y la urografía intravenosa han sido incluidas en la evaluación diagnóstica de estos pacientes, al informarnos de signos indirectos de ocupación del retroperitoneo (escoliosis antiálgica, mala definición de la silueta renal, masa con densidad de partes blandas que desplaza riñón o borra la línea del psoas). Sin embargo, actualmente estas pruebas radiológicas han sido desplazadas por la ecografía abdominal y sobre todo la TC, en el diagnóstico inmediato de la HRE. La ecografía es una exploración rápida, sencilla, no invasiva y de elevada sensibilidad, por lo que será de gran utilidad en el diagnóstico inicial de esta patología. No obstante, resulta menos eficaz a la hora de definir la naturaleza del proceso, ya que en ocasiones no es capaz de localizar el origen del sangrado ni la causa del mismo ${ }^{19,22}$. Esto coincide con los resultados de nuestra serie, donde la ecografía abdominal detectó el hematoma en el $81,8 \%$ de los casos, diagnosticando la causa del sangrado en tan sólo el 40,9\% de ellos.

En la actualidad, la TC se ha convertido en la prueba de imagen de elección en el diagnóstico de la HRE. Así, la hemorragia aguda se detectará como una lesión ocupante de espacio con un alto valor de atenuación (aproximadamente 70 unidades Hounsfield), que disminuirá en los días sucesivos. De la misma forma, es capaz de determinar el origen y la etiología del sangrado como los que ocasionan la existencia de tumores, al detectar densidades radiológicas propias de cada tejido, tales como el angiomiolipoma que presenta una densidad de -40 unidades Hounsfield, diferenciándolo del adenocarcinoma ${ }^{1,4}$. En aquellas situaciones de diagnóstico no aclarado por TC, podemos emplear otras pruebas como la RM o la arteriografía selectiva. En nuestro caso, la TC resultó ser la prueba más ventajosa, ya que detectó el hematoma en todos los casos y fue capaz de diagnosticar el origen del mismo en el $92,6 \%$ de las situaciones. En dos pacientes donde la TC no fue concluyente, recurrimos a la RM aportando un diagnóstico etiológico en ambos casos.

La arteriografia selectiva puede estar indicada en la evaluación de enfermedades vasculares asociadas a HRE, como panarteritis nodosa, aneurismas de arteria renal o malformaciones arteriovenosas, no detectadas por la $\mathrm{TC}^{15,23}$. Entre nuestros pacientes, la TC diagnosticó un aneurisma de arteria renal sin llegar a utilizar la arteriografía.

En caso de sangrado de origen suprarrenal, emplearemos técnicas diagnósticas específicas como la gammagrafía con metayodobencilguanidina (I-MIBG) para detectar la existencia de feocromocitomas o el test de ACTH o determinación de cortisol plasmático si se sospecha hemorragia suprarrenal bilateral ${ }^{3,7}$.

El manejo terapéutico de la HRE dependerá fundamentalmente de dos factores, la situación hemodinámica del paciente y la etiología del sangrado. En caso de inestabilidad hemodinámica 
iniciaremos medidas de reposición de la volemia mediante hemoderivados, coloides y cristaloides. Una vez conseguido estabilizar al paciente, realizaremos un estudio diagnóstico adecuado encaminado a determinar la etiología del proceso, basándonos principalmente en las pruebas radiológicas de imagen y en especial la TC. Si por el contrario, no se consigue la estabilidad hemodinámica del paciente o se inestabiliza en algún momento del proceso diagnóstico, será necesaria la exploración quirúrgica urgente y el tratamiento derivado.

En aquellos casos donde la TC detecte una masa renal adoptaremos una actitud más o menos conservadora en función de la naturaleza de la misma. Si se trata de un angiomiolipoma intentaremos ser lo más conservador posible optando por la embolización arterial selectiva, tumorectomía o nefrectomía parcial, si las condiciones lo permiten. Algunos autores ${ }^{12,24}$ recomiendan la obtención de biopsias intraoperatorias antes de proceder a cualquier cirugia conservadora, ante la posibilidad de coexistir otros tumores no detectados previamente. En nuestra serie, de 7 angiomiolipomas realizamos 2 embolizaciones arteriales al tratarse de tumores de 8 y $5 \mathrm{~cm}$. respectivamente, 1 tumorectomía bilateral y uno de los casos recibió tratamiento exclusivamente médico. Los 3 angiomiolipomas restantes se trataron mediante nefrectomía simple dada la inestabilidad hemodinámica de los pacientes. En caso de sospecha de adenocarcinoma, es mandatoria una nefrectomía radical, como realizamos en uno de nuestros pacientes. La rotura de un aneurisma de arteria renal suele requerir generalmente una cirugía de urgencia, siendo imposible en la mayoría de ocasiones preservar la unidad renal debido a la extensión del aneurisma al hilio renal haciendo impracticable la reparación ${ }^{3}$. Sin embargo, esto no aconteció en un caso que tuvimos de rotura de aneurisma de arteria renal, donde fue posible la reparación completa de la arteria renal sin necesitar la realización de una nefrectomía.

Si las pruebas de imagen detectan un tumor suprarrenal, la adrenalectomía parece ser la opción más aceptada, pero siempre que se haya descartado previamente la posibilidad de que se trate de un feocromocitoma. Si se confirma este diagnóstico, administraremos fármacos alfa-bloqueantes previamente a la cirugía ya que la secreción de catecolaminas por el tumor ocasiona una alta mortalidad intraoperatoria ${ }^{1,4}$. En uno de nuestros casos, realizamos una adrenalectomía por una metástasis suprarrenal de un carcinoma epidermoide de pulmón.

Las HRE de origen sistémico requerirán, en su mayoria, un tratamiento exclusivamente médico que variará según su etiología, siempre que no se evidencie patología asociada (tumoral, vascular,...). Así, pacientes con tratamientos anticoagulantes y/o antiagregantes plaquetarios o con discrasias sanguíneas precisarán de reposición sanguínea, plasma fresco, vitamina $\mathrm{K}$ o sulfato de protamina para controlar el sangrado, sin requerir intervención quirúrgica alguna. De la misma forma, las vasculitis (en especial la panarteritis nodosa) se tratarán de forma satisfactoria con corticoides e inmunosupresores, reservando la embolización arterial supraselectiva o la nefrectomía parcial para casos seleccionados ${ }^{1}$. En los 7 casos de origen sistémico que registramos, se practicó tratamiento médico en 6 (4 en tratamiento anticoagulante y 2 con discrasias sanguíneas en forma de mieloma múltiple y trombopenia idiopática), siendo necesaria la exploración quirúrgica con evacuación y drenaje del hematoma en el caso restante (terapia con anticoagulantes orales) dada su situación hemodinámica.

En conclusión, ante la sospecha de hemorragia retroperitoneal espontánea deberemos efectuar una TC abdomino-pélvica ya que es la prueba diagnóstica de elección (en nuestra serie, detectó el sangrado en el $100 \%$ de los casos, y la causa del mismo en más del 90\%). En cuanto a su manejo dependerá de la situación hemodinámica del paciente, de forma que si se consigue la estabilidad de la misma, el tratamiento inicialmente conservador de aquellas HRE de origen renal es la opción preferible por la mayoría de autores, entre ellos nosotros, reservando la cirugía para casos que no se consiga la estabilidad hemodinámica o con diagnóstico de tratamiento quirúrgico.

\section{REFERENCIAS}

1. Pode D, Caine M. Spontaneous retroperitoneal hemorrhage. J Urol. 1992; 147(2):311-318.

2. Suárez Artacho G, Rodríguez Muñoz J, Gómez Bravo MA, Campoy Martínez P, López Bernal F. Hematoma suprarrenal por rotura de mielolipoma. A propósito de un caso. Actas Urol Esp. 2004;28(10):785-788. 
3. Herranz Amo F, Verdú Tartajo F, Díez Cordero JM. Hemorragia retroperitoneal espontánea. En: Urología de Urgencias. Tema monográfico del LXI Congreso Nacional de Urología. Ene Ediciones, Madrid 1996; 179-184.

4. Machuca Santacruz J, Julve Villalta E, Galacho Bech A, Pérez Rodríguez D, Quiñonero Díaz A, Alonso Borrego JM, et al. Hematoma retroperitoneal espontáneo: nuestra experiencia. Actas Urol Esp. 1999;23(1):43-50.

5. Ivascu FA, Janczyk RJ, Bair HA, Bendick PJ, Howells GA. Spontaneous retroperitoneal hemorrhage. Am J Surg. 2005; 189(3):345-347.

6. Peña Porta JM, Pernaute Lavilla R. Síndrome de Wünderlich en paciente en hemodiálisis con enfermedad quística renal adquirida. Aportación de un nuevo caso. Actas Urol Esp. 1999;23(1):76-78.

7. García Rodríguez J, Fernández Gómez JM, Rodríguez Martínez JJ, Rodríguez Faba O, Regadera Sejas J, Escaf Bramada S. Hemorragia retroperitoneal espontánea por feocromocitoma. Arch Esp Urol. 2002;55(8):955-958.

8. Albi G, Del Campo L, Tagarro D. Wünderlich's syndrome: causes, diagnosis and radiological management. Clin Radiol. 2002;57(9):840-845.

9. Muro Bidaurre I, Sanz Jaka JP, Recarte Barriola JA, Martín López A, Hernaez Manrique I. Hemorragia retroperitoneal espontánea. Arch Esp Urol. 1999;52(3):274278.

10. Ara Goñi MA, Lapuente Arruebo MJ, Sanz Velez JI, Uribe Comps F, Vera Alvarez J, Esclarin Duna MA et al. Síndrome de Wünderlich: a proposito de tres casos. Actas Urol Esp. 1997;21(2):158-162.

11. Alonso A, Domínguez F, Verez M, Rodríguez B, Benavente J, Barros JM et al. Síndrome de Wúnderlich por metástasis de sarcoma gástrico en riñón. Aportación de un caso. Actas Urol Esp. 1996;20(4):395-398.

12. Vesga Molina F, Albisu Tristan A, Blasco De Villalonga M, Llerena Ibargurren R, Pertusa Peña C. Hemorragia retroperitoneal espontánea. Arch Esp Urol. 1994;47(2):129132 .

13. McDougal WS, Kursh ED, Persky L. Spontaneous rupture of the kidney with perirenal hematoma. J Urol. 1975;114 (2):181-184.

14. Cinman AC, Farrer J, Kaufman JJ. Spontaneous perinephric hemorrhage in a 65-year-old-man. J Urol. 1985; 133(5):829-832.
15. Belville JS, Morgentaler A, Loughlin KR, Tumeh SS Spontaneous perinephric and subcapsular hemorrhage: evaluation with CT, US and angiography. Radiology. 1989; 172(3):733-738.

16. Novicki DE, Turlington JT, Ball TP Jr. The evaluation and management of spontaneous perirenal hemorrhage. J Urol. 1980;123(5):764-765.

17. Mouded IM, Tolia BM, Bernie JE, Newman HR. Symptomatic renal angiomyolipoma: report of 8 cases, 2 with spontaneous rupture. J Urol. 1978;119(5):684-688.

18. Oesterling JE, Fishman EK, Goldman SM, Marshall FF. The management of renal angiomyolipoma. J Urol. 1986; 135(6): 1121-1124.

19. Brkovic D, Moehring K, Doersam J, Pomer S, Kaelble T, Riedasch G et al. Aetiology, diagnosis and management of spontaneous perirenal haematomas. Eur Urol. 1996;29(3): 302-307.

20. Reig Ruiz C, Morote Robles J, Saenz De Cabezón Martí J, Vila Barja J, López Pacios MA, De Torres Mateos JA et al. Hematoma perirrenal espontáneo. Arch Esp Urol. 1992;45 (7):615-619.

21. Ruiz Cerdá JL, Osca García JM, La Cruz Rodrigo J, Blanes Julia M, Server Pastor G, Jiménez Cruz JF. Hematoma perirrenal bilateral espontáneo como complicación de una panarteritis nodosa en un paciente con infección por el virus de inmunodeficiencia humano (HIV). Actas Urol Esp. 1993;17(3):196-198.

22. Zagoria RJ, Dyer RB, Assimos DG, Scharling ES, Quinn $\mathrm{SF}$. Spontaneous perinephric hemorrhage: imaging and management. J Urol. 1991;145(3):468-471.

23. Kendall A, Senay B, Coll M. Spontaneous subcapsular renal hematoma: diagnosis and management. J Urol.1988; 139(2):246-250.

24. Jardin A, Richard F, Le Duc A, Chatelain C, Le Guillou M, Fourcade $\mathrm{R}$ et al. Diagnosis and treatment of renal angiomyolipoma (based on 15 cases). Arguments in favor of conservative surgery (based on 8 cases). Eur Urol. 1980; 6(2):69-82.

Correspondencia autor: Dr. V. Gimeno Argente Servicio de Urología Hospital Universitario La Fe. Avda. Campanar, 21 - 46009 Valencia - Tel.: 963862700 E-mail autor: vigiar71@hotmail.com Información artículo: Original - Patología retroperitoneal Trabajo recibido: noviembre 2006

Trabajo aceptado: enero 2007 